\title{
Transformasi Fourier Fraksional dari Fungsi Gaussian
}

\author{
${ }^{1}$ Iin Sutrisna, ${ }^{2}$ Asriadi Nasrun, ${ }^{3}$ Mawardi Bahri, ${ }^{4}$ Syamsuddin Toaha
}

\begin{abstract}
The fractional Fourier transform is one of the generalizations of ordinary Fourier transform that depend on a particular angle $\theta$. In this paper we will derive the fractional Fourier transforms of a function that is well known in the field of analysis, namely Gaussian function.
\end{abstract}

Keywords: Fractional Fourier transform, Fourier transform, Gaussian Function.

\begin{abstract}
Abstrak
Transformasi Fourier fraksional adalah salah satu dari generalisasi dari transformasi Fourier biasa yang tergantung dari sudut $\theta$ tertentu. Dalam tulisan ini akan diungkapkan bentuk transformasi Fourier fraksional dari sebuah fungsi yang sangat dikenal di bidang analisis yaitu fungsi Gaussian.
\end{abstract}

Kata kunci: Transformasi Fourier fraksional, transformasi Fourier, fungsi Gaussian .

\section{Pendahuluan}

Transformasi Transformasi Fourier fraksional adalah sebuah generalisasi dari transformasi Fourier biasa dengan sebuah parameter orde $\theta$. Secara matematis, transformasi Fourier berorde $\theta$ adalah adalah sebuah pangkat $\theta$ dari operator transformasi Fourier. $\theta=\frac{\pi}{2}$ adalah orde transformasi Fourier fraksional dari transformasi Fourier biasa. Dengan perkembangan dari transformasi Fourier fraksional dan konsep yang terkait, domain frekuensi biasa hanya sebuah kasus khusus dari rangkaian kesatuan domain Fourier fraksional, dan kini telah sampai pada teori yang lebih umum sebagai alternatif untuk representasi signal. Setiap sifat dan aplikasi dari transformasi Fourier merupakan sebuah kasus khusus dari transformasi Fourier fraksional. Dalam semua area dimana konsep transformasi Fourier dan domain frekuensi digunakan, terdapat potensi untuk melakukan generalisasi dan perbaikan dengan menggunakan transformasi Fourier fraksional. Misalnya, telah diketahui dengan jelas hasil yang menyatakan bahwa pola diffraksi dari sebuah celah adalah dalam bentuk transformasi Fourier pola diffraksi celah tersebut, dapat digeneralisasi bahwa dalam jarak yang sangat dekat adalah transformasi Fourier fraksional dari celah [7]. Ada beberapa transformasi digeneralisasi menggunakan transformasi Fourier fraksional dan transformasi linear kanonik seperti seperti transformasi wavelet fraksional [1] dan distribusi Wigner-Ville berkaitan dengan transformasi linear kanonik [3].

\section{Transformasi Fourier dan Sifat-sifat Dasarnya}

\section{1,2,3,4, Program Studi Pascasarjana(S2) Matematika, FMIPA}

Universitas Hasanuddin,

Emai address: ${ }^{1}$ iinsutrisna3@gmail.com, ${ }^{2}$ asriadiasrunmath@gmail.com,

${ }^{3}$ mawardibahri@gmail.com, ${ }^{4}$ syamsuddint@yahoo.com 
Misalkan $f$ adalah fungsi terintegral Lebesgue pada $\mathbb{R}$, dinotasikan $f \in L^{1}(\mathbb{R})$, yakni semua fungsi bernilai kompleks $f$ yang memenuhi $\int_{-\infty}^{\infty}|f(t)| d t<\infty$. Karena $e^{-i \omega x}$ kontinyu dan terbatas, maka perkalian $e^{-i \omega x} f(x)$ terintegral lokal untuk setiap $\omega \in \mathbb{R}$. Juga, $\left|e^{-i \omega x}\right| \leq 1$ untuk semua $\omega$ dan $x$ di $\mathbb{R}$. Pandang integral

$$
\left\langle f, e^{-i \omega x}\right\rangle:=\int_{-\infty}^{\infty} f(x) e^{-i \omega x} d x, \quad \omega \in \mathbb{R} .
$$

Jelas bahwa

$$
\left|\int_{-\infty}^{\infty} f(x) e^{-i \omega x} d x\right| \leq\left|\int_{-\infty}^{\infty} f(x) d x\right| \leq \int_{-\infty}^{\infty}|f(x)| d x<\infty .
$$

Hal ini menyatakan bahwa integral ada untuk semua $\omega \in \mathbb{R}$.

Definisi 1.1 [1] Transformasi Fourier (TF) dari sebuah fungsi $f \in L^{1}(\mathbb{R})$ dituliskan sebagai

$$
\hat{f}(\omega)=\mathcal{F}\{f\}(\omega)=\frac{1}{\sqrt{2 \pi}} \int_{-\infty}^{\infty} f(x) e^{-i x \omega} d x .
$$

Generalisasi transformasi Fourier dalam matriks disebut dengan transformasi Fourier matriks bisa diperoleh di [4].

\section{Contoh 1.1}

Diberikan fungsi Gaussian $f(t)=e^{-t^{2}}$ maka

$$
\mathcal{F}\left\{e^{-t^{2}}\right\}(\omega)=\sqrt{\pi} e^{\left(-\frac{\omega^{2}}{4}\right)} .
$$

Misalkan $f(t) \in L^{1}(\mathbb{R})$ maka operator translasi, modulasi dan dilatasi dari fungsi $f$ berturut-turut dinyatakan sebagai berikut

$$
T_{a} f(t)=f(t-a), \quad M_{b} f(t)=e^{i b t} f(t), \quad D_{c} f(t)=\frac{1}{\sqrt{|c|}} f\left(\frac{t}{c}\right) .
$$

Teorema 1.1 [5]

Jika $f(t) \in L^{1}(\mathbb{R})$ maka berlaku

Teorema $1.2[5]$

$$
\begin{gathered}
\mathcal{F}\left\{T_{a} f(t)\right\}(\omega)=M_{-a} \hat{f}(\omega), \\
\mathcal{F}\left\{D_{a} f(t)\right\}(\omega)=D_{\frac{1}{a}} \hat{f}(\omega), \\
\mathcal{F}\left\{\overline{\left.D_{-1} f(t)\right\}(\omega)=\hat{f}(\omega),}\right. \\
\mathcal{F}\left\{M_{a} f(t)\right\}(\omega)=T_{a} \hat{f}(\omega) .
\end{gathered}
$$

Jika $f(t), t^{n} f(t) \in L^{1}(\mathbb{R})$ untuk bilangan asli $n$ maka $\frac{d^{n} \hat{f}(\omega)}{d \omega^{n}}$ ada dan berlaku

\section{Teorema 1.3 [5]}

$$
\frac{d^{n} \hat{f}(\omega)}{d \omega^{n}}=(-i)^{n} \mathcal{F}\left\{t^{n} f(t)\right\}(\omega)
$$

Jika $f(t), \frac{d f(t)}{d t}, \frac{d^{2} f(t)}{d t^{2}}, \ldots, \frac{d^{n} f(t)}{d t^{n}} \in L^{1}(\mathbb{R})$ untuk bilangan asli $n$ dan $\lim _{|t| \rightarrow \infty} \frac{d^{r} f(t)}{d t^{r}}=0$ untuk $r \in\{1,2, \ldots, n-1\}$ maka $\frac{d^{n} \hat{f}(\omega)}{d \omega^{n}}$ ada dan berlaku

Teorema 1.4 (Invers TF). ([5]

$$
\mathcal{F}\left\{\frac{d^{n} f(t)}{d t^{n}}\right\}(\omega)=(i \omega)^{n} \hat{f}(\omega)
$$

Jika $\hat{f}(\omega) \in L^{1}(\mathbb{R})$ maka berlaku

$$
f(t)=\mathcal{F}^{-1}\{\hat{f}(\omega)\}(t)=\frac{1}{\sqrt{2 \pi}} \int_{-\infty}^{\infty} \hat{f}(\omega) e^{i t \omega} d \omega .
$$




\section{Transformasi Fourier Fraksional}

Pada bagian ini akan mengungkap sifat-sifat dasar dari transformasi Fourier fraksional dan kemudian akan dibuktikan secara detail transformasi Fourier fraksional dari fungsi Gaussian.

Definisi 3.1 [6] Transformasi Fourier fraksional dengan sudut parameter $\theta$ dari sebuah fungsi $\phi(t) \in L^{1}(\mathbb{R})$, dinotasikan $\left(\mathcal{F}^{\theta} \phi\right)(\omega)=\hat{\phi}^{\theta}(\omega)$, dinyatakan sebagai

$$
\left(\mathcal{F}^{\theta} \phi(t)\right)(\omega)=\hat{\phi}^{\theta}(\omega)=\int_{-\infty}^{\infty} K^{\theta}(t, \omega) \phi(t) d t
$$

dengan kernelnya adalah

$$
K^{\theta}(t, \omega)=\left\{\begin{array}{lll}
C^{\theta} e^{i\left(t^{2}+\omega^{2}\right) \frac{\cot \theta}{2}-i t \omega \csc \theta}, & \frac{\pi}{2} \neq \theta \neq n \pi, \\
\frac{1}{\sqrt{2 \pi}} e^{-i t \omega}, & \theta=\frac{\pi}{2}, \\
\delta(t-\omega), & \theta=2 n \pi, \\
\delta(t+\omega), & \theta=(2 n+1) \pi, \quad n \in \mathbb{Z}
\end{array}\right.
$$

$\operatorname{dan} C^{\theta}=(2 \pi i \sin \theta)^{-\frac{1}{2}} e^{\frac{i \theta}{2}}=\sqrt{\frac{1-i \cot \theta}{2 \pi}}$.

Rumus invers yang berkorespondensi dengan transformasi Fourier fraksional untuk $\frac{\pi}{2} \neq \theta \neq n \pi$ dinyatakan sebagai

$$
\phi(t)=\int_{-\infty}^{\infty} \overline{K^{\theta}(t, \omega)} \hat{\phi}^{\theta}(\omega) d t
$$

dimana

$$
\overline{K^{\theta}(t, \omega)}=\overline{C^{\theta}} e^{-i\left(t^{2}+\omega^{2}\right) \frac{\cot \theta}{2}+i t \omega \csc \theta}=K^{-\theta}(t, \omega)
$$

dan

$$
\overline{C^{\theta}}=C^{-\theta} .
$$

Teorema 3.1 [6] Jika $\phi \in L^{1}(\mathbb{R})$ maka untuk $\frac{\pi}{2} \neq \theta \neq n \pi$ maka berlaku

$$
\mathcal{F}^{\theta}\left[\frac{d^{n}}{d t^{n}} \phi(t)\right](\omega)=\left(i \omega \sin \theta+\cos \theta \frac{d}{d \omega}\right)^{n} \mathcal{F}^{\theta}[\phi(t)](\omega)
$$

Definisi 3.2 Ruang Schwartz $S$ dan ruang $S c h w a r t z S_{\theta}$ yang berkaitan dengan TFF berturutturut didefinisikan sebagai

$$
\begin{aligned}
S & =\left\{\phi(t) \in C^{+\infty}(\mathbb{R})\left|\sup _{t \in \mathbb{R}}\right| t^{m} \frac{d^{n}}{d t^{n}} \phi(t) \mid<\infty, \forall m, n \in \mathbb{N}\right\} \\
S_{\theta} & =\left\{\tau(t) \in C^{+\infty}(\mathbb{R})\left|\sup _{t \in \mathbb{R}}\right| t^{m} \Delta_{t}^{n} \tau(t) \mid<\infty, \forall m, n \in \mathbb{N}\right\}
\end{aligned}
$$

dimana $\Delta_{t}=\left(\frac{d}{d t}-i t \cot \theta\right)$.

Teorema 3.2 Misalkan $K^{\theta}(t, \omega)$ adalah kernel dari transformasi Fourier fraksional dan $\Delta_{t}=\left(\frac{d}{d t}-i t \cot \theta\right) . M a k a$

(i) $\quad \Delta_{t}^{n} K^{\theta}(t, \omega)=(-i \omega \csc \theta)^{n} K^{\theta}(t, \omega), \forall n \in \square \cup\{0\}$,

(ii) $\mathcal{F}^{\theta}\left[\left(\Delta_{t}^{*}\right)^{n} \phi(t)\right](\omega)=(-i \omega \csc \theta)^{n} \mathcal{F}^{\theta}[\phi(t)](\omega), \quad \forall n \in \square \cup\{0\}$ dan $\phi \in S$,

dengan $\Delta_{t}^{*}=-\left(\frac{d}{d t}+i t \cot \theta\right)$. 
Iin Sutrisna, Asriadi Nasrun, Mawardi Bahri, Syamsuddin Toaha

Teorema 3.3 Jika $\phi \in L^{1}(\mathbb{R})$, maka $\hat{\phi}^{\theta}$ memenuhi kondisi berikut

(i) $\quad \hat{\phi}^{\theta} \in L^{\infty}(\mathbb{R})$ dengan $\left\|\hat{\phi}^{\theta}\right\|_{\infty} \leq\left|C^{\theta}\right|\left\|\hat{\phi}^{\theta}\right\|_{1}$,

(ii) $\hat{\phi}^{\theta}$ kontinyu pada $\mathbb{R}$,

(iii) $\quad \hat{\phi}^{\theta}(\omega) \rightarrow 0$ untuk $\omega \rightarrow+\infty$ atau $\omega \rightarrow-\infty$.

Teorema 3.4 Jika $\hat{\phi}^{\theta}$ dan $\hat{\varphi}^{\theta}$ berturut-turut adalah transformasi Fourier fraksional dari $\phi, \varphi \in L^{1}(\mathbb{R}), m a k a$

$$
\begin{aligned}
\int_{-\infty}^{\infty} \phi(t) \overline{\varphi(t)} d t & =\int_{-\infty}^{\infty} \hat{\phi}^{\theta}(\omega) \overline{\hat{\varphi}^{\theta}(\omega)} d \omega \\
\int_{-\infty}^{\infty} \hat{\phi}^{\theta}(t) \varphi(t) d t & =\int_{-\infty}^{\infty} \phi(\omega) \hat{\varphi}^{\theta}(\omega) d \omega
\end{aligned}
$$

dan

$$
\int_{-\infty}^{\infty}|\phi(t)|^{2} d t=\int_{-\infty}^{\infty}\left|\hat{\phi}^{\theta}(\omega)\right|^{2} d \omega
$$

Teorema berikut mengungkapkan transformasi Fourier fraksional dari sebuah fungsi Gaussian yang merupakan hasil utama dari tulisan ini.

Teorema 3.5 (TFF fungsi Gaussian). Jika $\phi \in L^{1}(\mathbb{R}), \frac{\pi}{2} \neq \theta \neq m \pi, m \in \mathbb{Z}$ maka untuk konstanta real a $>0$ berlaku

$$
\left(\mathcal{F}^{\theta}\left(e^{-a t^{2}}\right)\right)(\omega)=\sqrt{\frac{1-i \cot \theta}{2 a-i \cot \theta}} e^{\left(\frac{i}{2} \omega^{2} \cot \theta-\frac{(\omega \csc \theta)^{2}}{2(2 a-i \cot \theta)}\right)} .
$$

Bukti:

Perhatikan bahwa, berdasarkan definisi diperoleh $\left(\mathcal{F}^{\theta}\left(e^{-a t^{2}}\right)\right)(\omega)$

$=C^{\theta} \int_{-\infty}^{\infty}\left(e^{i\left(t^{2}+\omega^{2}\right) \frac{\cot \theta}{2}-i t \omega \csc \theta}\right) e^{-a t^{2}} d t$

$=\sqrt{\frac{1-i \cot \theta}{2 \pi}} \int_{-\infty}^{\infty} e^{i\left(t^{2}+\omega^{2}\right) \frac{\cot \theta}{2}-a t^{2}-i t \omega \csc \theta} d t$

$=\sqrt{\frac{1-i \cot \theta}{2 \pi}} \int_{-\infty}^{\infty} e^{i\left(t^{2}+\omega^{2}\right) \frac{\cot \theta}{2}-a t^{2}-i t \omega \csc \theta} d t$

$=\sqrt{\frac{1-i \cot \theta}{2 \pi}} \int_{-\infty}^{\infty} e^{\frac{i \cot \theta}{2} t^{2}+\frac{i \cot \theta}{2} \omega^{2}-a t^{2}-i t \omega \csc \theta} d t$

$=\sqrt{\frac{1-i \cot \theta}{2 \pi}} \int_{-\infty}^{\infty} e^{\frac{i \cot \theta}{2} \omega^{2}} e^{\left(\frac{i \cot \theta}{2}-a\right) t^{2}-i t \omega \csc \theta} d t$

$=\sqrt{\frac{1-i \cot \theta}{2 \pi}} e^{\frac{i \cot \theta}{2} \omega^{2}} \int_{-\infty}^{\infty} e^{\left(\frac{i \cot \theta}{2}-a\right) t^{2}-i t \omega \csc \theta} d t$

$=\sqrt{\frac{1-i \cot \theta}{2 \pi}} e^{\frac{i \cot \theta}{2} \omega^{2}} \int_{-\infty}^{\infty} e^{\left(\frac{-2 a+i \cot \theta}{2}\right) t^{2}-i t \omega \csc \theta} d t$

$=\sqrt{\frac{1-i \cot \theta}{2 \pi}} e^{\frac{i \cot \theta}{2} \omega^{2}} \int_{-\infty}^{\infty} e^{\left(\frac{-2 a+i \cot \theta}{2}\right)\left(t^{2}-i\left(\frac{2}{-2 a+i \cot \theta}\right) t \omega \csc \theta\right)} d t$ 


$$
\begin{aligned}
& =\sqrt{\frac{1-i \cot \theta}{2 \pi}} e^{\frac{i \cot \theta}{2} \omega^{2}} \int_{-\infty}^{\infty} e^{\left(\frac{-2 a+i \cot \theta}{2}\right)\left(t^{2}-\left(\frac{2}{2 a i+\cot \theta}\right) t \omega \csc \theta\right)} d t \\
& =\sqrt{\frac{1-i \cot \theta}{2 \pi}} e^{\frac{i \cot \theta}{2} \omega^{2}} \int_{-\infty}^{\infty} e^{\left(\frac{-2 a+i \cot \theta}{2}\right)\left(t^{2}-\left(\frac{2 \omega \csc \theta}{2 a i+\cot \theta}\right) t\right)} d t \\
& =\sqrt{\frac{1-i \cot \theta}{2 \pi}} e^{\frac{i \cot \theta}{2} \omega^{2}} \int_{-\infty}^{\infty} e^{\left(\frac{-2 a+i \cot \theta}{2}\right)\left(\left(t-\frac{\omega \csc \theta}{2 a i+\cot \theta}\right)^{2}-\left(\frac{\omega \csc \theta}{2 a i+\cot \theta}\right)^{2}\right)} d t \\
& =\sqrt{\frac{1-i \cot \theta}{2 \pi}} e^{\frac{i \cot \theta}{2} \omega^{2}} \int_{-\infty}^{\infty} e^{\left(\frac{-2 a+i \cot \theta}{2}\right)\left(t-\frac{\omega \csc \theta}{2 a i+\cot \theta}\right)^{2}-\left(\frac{-2 a+i \cot \theta}{2}\right)\left(\frac{\omega \csc \theta}{2 a i+\cot \theta}\right)^{2}} d t \\
& =\sqrt{\frac{1-i \cot \theta}{2 \pi}} e^{\frac{i \cot \theta}{2} \omega^{2}} e^{-\left(\frac{-2 a+i \cot \theta}{2}\right)\left(\frac{\omega \csc \theta}{2 a i+\cot \theta}\right)^{2}} \int_{-\infty}^{\infty} e^{\left(\frac{-2 a+i \cot \theta}{2}\right)\left(t-\frac{\omega \csc \theta}{2 a i+\cot \theta}\right)^{2}} d t \\
& =\sqrt{\frac{1-i \cot \theta}{2 \pi}} e^{\frac{i \cot \theta}{2} \omega^{2}} e^{-i\left(\frac{2 a i+\cot \theta}{2}\right)\left(\frac{\omega \csc \theta}{2 a i+\cot \theta}\right)^{2}} \int_{-\infty}^{\infty} e^{-\left(\frac{2 a-i \cot \theta}{2}\right)\left(t-\frac{\omega \csc \theta}{2 a i+\cot \theta}\right)^{2}} d t
\end{aligned}
$$

Karena integral Gaussian $\int_{-\infty}^{\infty} e^{-k x^{2}} d x=\sqrt{\frac{\pi}{k}}$ maka

$$
\begin{aligned}
& \left(\mathcal{F}^{\theta}\left(e^{-a t^{2}}\right)\right)(\omega) \\
& =\sqrt{\frac{1-i \cot \theta}{2 \pi}} e^{\frac{i \cot \theta}{2} \omega^{2}} e^{-i\left(\frac{\omega^{2} \csc ^{2} \theta}{2(2 a i+\cot \theta)}\right)} \sqrt{\frac{\pi}{\left(\frac{2 a-i \cot \theta}{2}\right)}} \\
& =\sqrt{\frac{1-i \cot \theta}{2 \pi}} e^{\frac{i \cot \theta}{2} \omega^{2}} e^{-i\left(\frac{\omega^{2} \csc ^{2} \theta}{2(2 a i+\cot \theta)}\right)} \sqrt{\frac{2 \pi}{2 a-i \cot \theta}} \\
& =\sqrt{\frac{1-i \cot \theta}{2 a-i \cot \theta}} e^{\left(\frac{i}{2} \omega^{2} \cot \theta-\frac{(\omega \csc \theta)^{2}}{2(2 a-i \cot \theta)}\right)}
\end{aligned}
$$

\section{Kesimpulan}

Dengan telah diungkapkannya bentuk TFF dari fungsi Gaussian yaitu

$$
\left(\mathcal{F}^{\theta}\left(e^{-a t^{2}}\right)\right)(\omega)=\sqrt{\frac{1-i \cot \theta}{2 a-i \cot \theta}} e^{\left(\frac{i}{2} \omega^{2} \cot \theta-\frac{(\omega \csc \theta)^{2}}{2(2 a-i \cot \theta)}\right)}
$$

diharapkan telah memudahkan mencari bentuk TFF dari fungsi lain yang melibatkan fungsi Gaussian.

\section{Daftar Pustaka}

[1] Bahri M. dan Ashino R., 2017. Logarithmic Uncertainty Principles Convolution Theorem Related to Continious Fractional Wavelet Transform and its Properties on a Generalized Sobolev Space. International Journal of wavelet, Multiresolution and Information Processing. Vol. 15, No.5, 
Iin Sutrisna, Asriadi Nasrun, Mawardi Bahri, Syamsuddin Toaha

[2] Bahri M. dan Ashino R. dan Amir A.K., 2017. Correlation Formulation Using Relationship Between Convolution and Correlation in Linear Canonical Transform Domain. International Conference on Wavelet Analysis and Pattern Recognition, Ningbo, China., hal. 177-182.

[3] Bahri M. dan Ashino R., 2015. Convolution and Correlation Theorem for Wigner-Ville Distribution Associated with Linear Canonical Transform. Intenational Conference on Information Technology-New Generations (ITNG), hal. 341-346.

[4] Bahri M., 2010. Consruction of Quaternion-Valued Wavelets. Matematika: Malaysian Journal of Industrial and Applied Mathematics. Vol. 26, No.1, hal. 107-114.

[5] Clarke, B.M.N., 2009. Fourier Theory. Departement of Mathematics, Macquarie University, hal. 1-65.

[6] Akhilesh P., Manna S.,. Ashutosh Mahato dan V.K. Singh., 2014. The generalized continuous wavelet transform associated with the fractional Fourier transform. Journal of Computational and Applied Mathematics., hal. 1-12.

[7] Ozaktas H. dan Kutay A., 1996. Introduction to fraksional Fourier transform and its application., hal. 3-4. 